\title{
Widening the scope of diversity
}

\author{
An overlooked bias in research practices and current global events call for a broader approach to addressing issues \\ of diversity.
}

T he neuroscience community has tackled issues of diversity in various ways. Anne's List (https://anneslist. net/) and Women in Neuroscience (https://www.winrepo.org/) list women neuroscientists categorized by expertise, career status and geographic location. The PowerHour at Gordon Research Conferences (https://www.grc.org/ the-power-hour/) provides a safe space for attendees to discuss persistent and pervasive issues of inequity. Diversity and inclusion initiatives are a part of many neuroscience societies and organizations. BiasWatchNeuro (https://biaswatchneuro.com/) tracks the gender ratios of speakers at neuroscience meetings and of authors in neuroscience journals and compares them to the base rates of women in relevant subfields.

Journals have also examined gender diversity among published authors and reviewers. Nature Neuroscience reported the percentages of female authors of primary research articles and the gender composition of our reviewer pool in $2005^{1}$ and $2016^{2}$. In response to the 2016 findings we have, since $2017^{2}$, made a concerted effort to improve the gender ratio among the scientists we invite to review and write commissioned pieces.

Yet a study from Dworkin and colleagues $^{3}$ highlights one aspect that has been overlooked: gender diversity in citation practices. The study shows that from 2009-2018, papers with a female researcher as first or last author were cited less often than papers with male first and last authors in five neuroscience journals, including Nature Neuroscience. Author groups with male researchers as both first and last authors overcited papers with first and last male authors (MM) and undercited papers with woman scientists as first and/or last author (WM, MW, WW). In contrast, groups with women as first and last authors undercited MM and WM papers, overcited MW papers and overcited WW papers at nearly half the rate than MM author groups undercite these papers. Although the number of women neuroscientists increased over this period, the undercitation of women-authored papers worsened, particularly by male-led author groups. These observations suggest that men are less likely to cite research led by women.

We were unaware that biased citation behavior occurred in our pages, and we recognize that this aspect of gender inequality will be disconcerting to our community. While reference limits could play a potential role, the preference for citing male-authored works has persisted despite an increase in the relative proportions of female-authored works over the years. Enabling authors to cite more papers may not be enough to redress the problem so long as such practices remain unchecked. We must acknowledge that citation practices are yet another instance where implicit bias can influence behavior. So how can we begin to address this imbalance?

For authors writing a manuscript, be mindful when deciding which papers to cite. Consider which papers best support your statements and conclusions. Challenge yourself to look beyond the last paper you read, several related works from a single group in the field, and papers authored by researchers within your professional network. Once you have assembled your reference list, review it and look for evidence of implicit bias. Aspire for an equal gender ratio in your citations. Similarly, as you prepare your list of reviewer suggestions, examine whether it is solely based on the papers you have cited. Suggest a diverse pool of researchers that is representative of the current composition of the community.

As a reviewer, if you cannot commit to peer review, suggest alternate reviewers and assess whether the suggested list is balanced in terms of gender. This will help diversify our pool of reviewers, whose indispensable input influence our editorial decisions and shape the published paper. As you review a manuscript, evaluate whether the citations include the most appropriate papers and whether their authors represent the evolving gender ratio in the field.

The Nature Neuroscience editorial team strives to be impartial with all our decisions, but despite gender parity on the team, we are not free of implicit bias. Dworkin and colleagues show that the gender ratio in authorship has not changed considerably in our pages across a 20 -year $\operatorname{span}^{3}$. We pledge to continue working to improve this. Editors are susceptible to same-gender preference in selecting reviewers ${ }^{4}$, and we will maintain our efforts to improve the diversity of the reviewers we invite, bolstered by your suggestions. We will also endeavor to invite a diverse set of speakers when organizing conferences.

As we write this piece in the midst of the COVID-19 pandemic, it is impossible to ignore the global, thunderous cry for racial justice and equity in light of the recent killings of George Floyd, Breonna Taylor, Ahmaud Arbery and too many others. Diversity in neuroscience cannot focus on gender alone. It is, and always has been, an intersectional issue. We need to recognize that disparities arise with respect to gender, race, ethnicity, LGBTQ+ representation, disability, career status and geography, to name only a few aspects. Yet evidence that ongoing diversity efforts are effective ${ }^{5}$ perhaps can serve as a balm. Our community can improve gender ratio in citations. And as we do so, we must broaden our efforts to consider other facets of diversity. It is an enormous task, and we cannot always have an immediate impact in every aspect of our scientific lives. But as a community, we can work collectively and support each other as we gather information and develop more resources to educate ourselves and move toward equity for all. Nature Neuroscience asks our readers to suggest ways in which our team can better support diversity initiatives in neuroscience. We are here and we are listening.

Published online: 26 June 2020

https://doi.org/10.1038/s41593-020-0670-2

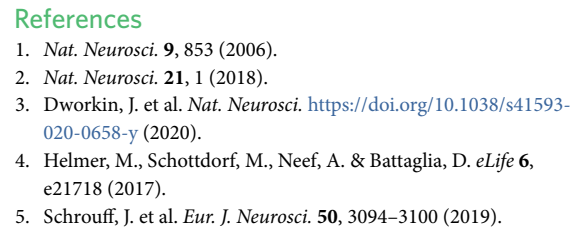

